\title{
Risk factors for recurrent Clostridium difficile infection (CDI) hospitalization among hospitalized patients with an initial CDI episode: a retrospective cohort study
}

\author{
Marya D Zilberberg ${ }^{1,2^{*}}$, Kimberly Reske ${ }^{3}$, Margaret Olsen $^{3},{\text { Yan } \text { Yan }^{3} \text { and Erik R Dubberke }}^{3}$
}

\begin{abstract}
Background: Recurrent Clostridium difficile infection (rCDI) is observed in up to $25 \%$ of patients with an initial CDI episode (iCDI). We assessed risk factors for rCDI among patients hospitalized with iCDI.

Methods: We performed a retrospective cohort study at Barnes-Jewish Hospital from 1/1/03 to 12/31/09. iCDI was defined as a positive toxin assay for $C$. difficile with no $C D I$ in previous 60 days, and rCDI as a repeat positive toxin $\leq 42$ days of stopping iCDI treatment. Three demographic, 13 chronic and 12 acute disease characteristics, and 21 processes of care were assessed for association with rCDI. Cox modeling identified independent risk factors for rCDI.

Results: 425 (10.1\%) of 4,200 patients enrolled developed rCDI. Of the eight risk factors for rCDI on multivariate analyses, the strongest three were 1) high-risk antimicrobials following completion of iCDI treatment (HR 2.95, 95\% Cl 2.25-3.86), 2) community-onset healthcare-associated iCDI (HR 1.80, 95\% Cl 1.41-2.29) and 3) fluoroquinolones after completion of iCDI treatment (HR 1.56, 95\% Cl 1.63-2.08). Other risk factors included gastric acid suppression, $\geq 2$ hospitalizations within prior 60 days, age, and IV vancomycin after iCDI treatment ended.

Conclusions: The rCDI rate was $10.1 \%$. Recognizing such modifiable risk factors as certain antimicrobial treatments and gastric acid suppression may help optimize prevention efforts.
\end{abstract}

Keywords: C. difficile, Risk factors, Recurrence

\section{Background}

Over the past decade Clostridium difficile infection (CDI) has increased in both frequency and severity in the US and abroad. A study from Quebec identified a 5-fold rise in the incidence of hospitalizations with CDI over 13 years, accompanied by a doubling in the risk of complicated disease [1]. Similarly, multiple US-based studies have reported a more-than-doubling of hospitalizations with a CDI diagnosis between 2000 and 2005 [2,3]. These numbers have continued to rise through 2009, albeit less rapidly [4]. Much of this growth is thought to be due to the recent emergence of the hypervirulent epidemic strain of C. difficile, BI/NAP1/027. A fluoroquinolone-resistant

\footnotetext{
* Correspondence: evimedgroup@gmail.com

'University of Massachusetts, Amherst, MA, USA

${ }^{2}$ EviMed Research Group, LLC, PO Box 303, Goshen, MA, USA

Full list of author information is available at the end of the article
}

toxin overproducer, this strain has now been detected in most of the states in the US, in North America, Europe and beyond [5].

One of the most challenging aspects of CDI is its propensity to recur. Both metronidazole and vancomycin, first-line therapies recommended in the joint evidencebased practice guideline from the Society of Healthcare Epidemiology of America (SHEA) and Infectious Diseases Society of America (IDSA), have exhibited unacceptably high rates of recurrence [6]. Indeed, a recent meta-analysis has found that CDI recurs in 13\% - 50\% of all patients after an initial episode, and in the setting of a randomized controlled trial, the recurrence rate was $25 \%$ [7-9].

Recurrent CDI (rCDI) is a cause of much morbidity, and its economic impact is likely substantial. Several studies have identified important risk factors for rCDI, 
including advanced age, chronic renal insufficiency, elevated white blood cell count, low serum albumin, use of proton pump inhibitors (PPI), and continued use of systemic antimicrobials during the initial CDI episode (iCDI) [7,10-13]. However, a meta-analysis identified major gaps in our understanding of the risk factors for CDI recurrence [7]. Although the authors found concomitant antimicrobials, gastric acid suppressants and older age to be strongly predictive of $\mathrm{rCDI}$, other factors, including iCDI treatment and specific non-CDI antimicrobials, could not be evaluated adequately due to the lack of robust data. Additionally, most studies have focused on the factors immediately preceding rCDI onset, ignoring the possibility that factors present at or near the onset of the iCDI episode may also impact this risk. In fact, recent data suggest that the burden of community-onset healthcare-facility associated (COHCFA) CDI is much higher than previously appreciated, and poses an additional risk pool for inpatient exposure $[14,15]$. Since CO-HCFA implies an ongoing exposure to the healthcare system, it may itself be a marker for a recurrence.

A precise understanding of who is likely to recur is an important clinical question for two reasons. First, if there are modifiable exposures that increase this risk, knowing what they are may aid clinicians in avoiding them. Second, if patient characteristics not subject to modification predispose to $\mathrm{rCDI}$, recognizing them may help target preventive measures more effectively. In order to define more fully the risk factors for $\mathrm{rCDI}$, we conducted a single center retrospective cohort analysis among patients hospitalized with an iCDI episode.

\section{Methods}

This study was approved by the Washington University Institutional Review Board, and its conduct was in compliance with the Helsinki Declaration.

\section{Cohort definition}

We conducted a retrospective cohort study of all adult (age $\geq 18$ years) patients with an inpatient episode of iCDI at Barnes-Jewish Hospital (BJH) between January 1, 2003, and December 31, 2009. An episode of CDI was defined as a positive toxin assay (C. DIFFICILE TOX A/B II from Techlab, Blacksburg, VA, USA) for C. difficile. Because the hospital laboratory performs a test for $C$. difficile only if the treating physician suspects CDI and if the stool is unformed, all patients with positive toxin results were considered to be CDI case patients. The first episode of CDI during the study period in the absence of any CDI in the prior 60 days was defined as the iCDI, and patients were included only once. Patients were excluded if they died during or were discharged to hospice from the iCDI hospitalization.
All included patients were followed for 42 days from the date of the end of iCDI treatment or until rCDI onset, defined as a repeat positive toxin within this time frame. Initial CDI cases were categorized according to published surveillance definitions as community-onset healthcare facility-associated (CO-HCFA) (indeterminate CDI cases were grouped with CO-HCFA), healthcare facility-onset (HCFO), and community-associated (CA) [16].

\section{Data sources}

Demographic and clinical data were derived from $\mathrm{BJH}$ Medical Informatics databases and the $\mathrm{BJH}$ electronic medical records. The data available from the Informatics databases included $C$. difficile toxin assay results and date of stool collection; patient demographics; dates of admission and discharge; discharge disposition; admission location; ICD-9-CM diagnosis (used to define underlying comorbidities in the year prior and during the index hospitalization) and procedure (assessed only during the index hospitalization) codes; dates of ICU stays; start and stop dates of all inpatient CDI treatments, gastric acid suppressors and antimicrobials; and white blood cell count, hemoglobin, serum creatinine, and serum albumin levels on admission and at the time of positive $C$. difficile toxin assays from the index admission and all readmissions in the 42 days after iCDI treatment end. The $\mathrm{BJH}$ medical records included data on antimicrobials and CDI treatments the patient received as an outpatient within the $\mathrm{BJH}$ system, and whether a readmission was for CDI. In addition, admission and discharge summaries were reviewed for all included hospitalizations to help determine whether the patient had a history of CDI at another healthcare facility or as an outpatient.

\section{Statistical analyses}

The exposure interval was divided into three periods: 1 ) from hospital admission until diagnosis of $\mathrm{iCDI}, 2$ ) from the time of diagnosis of iCDI until the end of its treatment, and 3) from the end of iCDI treatment until the onset of recurrence or until the end of the 42-day monitoring period for recurrence. We compared patients with rCDI to those without rCDI based on their characteristics in these time periods. Cox proportional hazards modeling was used to determine variables associated with at least one episode of rCDI on univariate analysis. Antimicrobials were categorized based on association with CDI as highrisk (cephalosporins, aminopenicillins, and clindamycin), low-risk (aminoglycosides, beta lactamase inhibitors, carbepenems, daptomycin, doxycycline, linezolid, macrolides, rifampin, rifaximin, and tigecycline), fluoroquinolones ( $>90 \%$ was ciprofloxacin), and intravenous vancomycin $[17,18]$. Gastric acid suppressors (histamine receptor 2 
blockers [HR2B] and proton pump inhibitors [PPI]), choice and duration of iCDI treatment, and iCDI severity, as defined by the SHEA/IDSA Clinical Practice Guidelines for CDI, were also assessed as potential risk factors for rCDI [6].

We employed extended Cox proportional hazards modeling to determine independent risk factors for at least one episode of rCDI, with variable selection according to the methodology of Hosmer-Lemeshow [19]. Variables eligible for inclusion in the multivariable models were those associated with increased risk of rCDI from the literature or those with clinical or biologic plausibility, and those with $\mathrm{p}$-values $<0.20$ in the univariate analyses. Antimicrobial exposures from the end of CDI treatment until rCDI or 42 days were analyzed as timedependent variables. Backward stepwise selection was used to arrive at the best-fitting and most parsimonious model. All relevant 2-way interactions were tested after selection of the main effects, and included in the final models only if they were significant at the alpha $\leq 0.05$. The proportional hazards assumption was verified by assessing the parallel nature of curves in log-log plots. The appropriate functional formats of continuous variables were determined by examining nonparametric regression (smoothing) plots with a restricted cubic spline function. To facilitate interpretation of results, the hazard ratios for the piecewise linear spline variable (fluoroquinolone exposure while on CDI treatment) compared the hazards of developing CDI for values between the 75 th and the 25th percentiles of the variable [20]. To assess the importance of time dependency for antimicrobial exposures that occur after CDI treatment, these exposures included in the final model were also analyzed in a time-independent fashion.

All analyses were performed in SAS version 9.3 (SAS Institute, Cary, NC) and R ( R Foundation, Vienna, Austria) [21]. All statistical testing was two-tailed with significance set at the alpha level $\leq 0.05$.

\section{Results}

Among the 4,200 patients enrolled with iCDI, 425 (10.1\%) had at least one rCDI identified. Those with a recurrence were older (median age 64.8, range 18.3 - 98.2, vs. 61.6, range 18.0 - 102.4), and had a greater comorbidity burden, as evidenced by the higher Charlson score than those without (Table 1). Of the specific comorbidities, diabetes mellitus was more prevalent in the rCDI group than in the group without rCDI. Notably, prior exposure to the healthcare system was more likely among those with rCDI than those without. Persons with rCDI were nearly twice as likely to fit the surveillance definition for CO-HCFA CDI during their initial episode (39\%) as those without rCDI $(22 \%, \mathrm{p}<0.001)$. Consistent with this, patients with rCDI had a higher frequency of at least one inpatient admission within 60 days prior to the iCDI episode (53\% vs. $39 \%, \mathrm{p}<0.001)$, as well as a higher risk for multiple recent hospitalizations than those without rCDI (Table 1). The iCDI admission laboratory data did not differ substantively between the groups.

There were several differences between patients with and those without rCDI with respect to exposure to medications known to raise the risk of CDI (Table 2). Nearly $3 / 4$ of all patients in each group were on at least one antimicrobial at the onset of their iCDI. There were no differences in antibiotics considered to be "high-risk" for causing CDI between the rCDI and non-rCDI groups. Conversely, antimicrobials designated as "lowrisk" for CDI were used with lower frequency in patients with (22\%) compared to those without $\mathrm{rCDI}(28 \%$, $\mathrm{p}=0.02)$. Fluoroquinolone treatment was more prevalent during the iCDI onset among those with rCDI (28\%) than those without $(23 \%, \mathrm{p}=0.02)$. Furthermore, patients with rCDI who used gastric acid suppressors were nearly twice as likely as those without to be started on one within 24 hours of the iCDI diagnosis (Table 2).

Although there were no differences in the treatment regimen aimed at the iCDI episode, patients who developed rCDI were more frequently started on such high-risk medications as gastric suppressors and non-CDI antimicrobials, regardless of their designation as high- or low-risk, after the onset of iCDI (Table 2). Those with subsequent rCDI were more likely to be discharged to a healthcare facility following the iCDI hospitalization than those without rCDI, though this difference did not reach statistical significance. Following the hospitalization with iCDI, patients with a subsequent rCDI were also more likely to be readmitted to the hospital both before and/or after the end of the iCDI treatment (Table 3).

In a multivariate Cox proportional hazards model, where we examined 49 potential covariates for their impact on the risk of rCDI, eight factors emerged as predictive of a future episode of rCDI (Table 4). In addition to age, case status of iCDI designation of CO-HCFA was strongly associated with rCDI, increasing its risk by $80 \%$ (HR 1.80, 95\% CI 1.41 to 2.29). In a similar vein, having had two or more inpatient hospitalization within 60 days prior to the onset of CDI was associated with an increased risk of rCDI ( $\mathrm{HR} 1.40,95 \%$ CI 1.04-1.89). A number of modifiable risk factors also predicted the risk of rCDI. They included initiation of gastric acid suppressors at the time of iCDI diagnosis (HR 1.36, 95\% CI 1.004 to 1.85 ), and cumulative exposure to fluoroquinolones while on iCDI therapy (HR 1.45, 95\% CI 1.09 to 1.41). Exposure to fluoroquinolones ( $\mathrm{HR} \mathrm{1.56,95 \%} \mathrm{CI}$ 1.16 to 2.08 ), IV vancomycin (HR $1.45,95 \%$ CI 1.09 to 1.92), and high-risk antimicrobials (HR 2.95, 95\% CI 2.25 to 3.86) at any time $t$ after the end of iCDI therapy ended also increased the risk of rCDI. Demonstrating 
Table 1 Patient characteristics and treatments at hospital admission involving the initial CDI episode

\begin{tabular}{|c|c|c|c|}
\hline Patient characteristics & $\begin{array}{l}\text { Patients who developed rCDI } \\
\qquad(\mathrm{n}=425)\end{array}$ & $\begin{array}{l}\text { Patients who did not develop rCDI } \\
\qquad(\mathrm{n}=3775)\end{array}$ & $\begin{array}{c}\text { Hazard ratio } \\
95 \% \mathrm{Cl}\end{array}$ \\
\hline \multicolumn{4}{|l|}{ Demographics } \\
\hline Age, years (median[range]) & $64.8(18.3-98.2)$ & $61.6(18.0-102.4)$ & $1.01(1.01-1.02)$ \\
\hline Gender: female & $210(49)$ & $1824(48)$ & $1.05(0.87-1.27)$ \\
\hline \multicolumn{4}{|l|}{ Comorbidities $^{a}$} \\
\hline Myocardial infarction & $40(9)$ & $328(9)$ & $1.12(0.81-1.55)$ \\
\hline Congestive heart failure & $108(25)$ & $854(23)$ & $1.23(0.99-1.53)$ \\
\hline Peripheral vascular disease & $34(8)$ & $269(7)$ & $1.13(0.79-1.60)$ \\
\hline Cerebrovascular disease & $41(10)$ & $256(7)$ & $1.51(1.10-2.09)$ \\
\hline Chronic renal failure & $21(5)$ & $190(5)$ & $0.98(0.64-1.53)$ \\
\hline Dementia & $5(1)$ & $23(1)$ & $1.83(0.76-4.41)$ \\
\hline Chronic obstructive pulmonary disease & $116(27)$ & $912(24)$ & $1.18(0.95-1.46)$ \\
\hline Rheumatologic disease & $18(4)$ & $146(4)$ & $1.11(0.69-1.78)$ \\
\hline Peptic ulcer disease & $20(5)$ & $154(4)$ & $1.18(0.75-1.85)$ \\
\hline Mild liver disease & $17(4)$ & $201(5)$ & $0.81(0.50-1.32)$ \\
\hline Moderate to severe liver disease & $12(3)$ & $134(4)$ & $0.86(0.48-1.53)$ \\
\hline Diabetes & $135(32)$ & $975(26)$ & $1.32(1.08-1.62)$ \\
\hline Paraplegia or hemiplegia & $12(3)$ & $78(2)$ & $1.40(0.79-2.45)$ \\
\hline Any malignancy (excluding leukemia/lymphoma) & $83(20)$ & $770(20)$ & $0.99(0.78-1.25)$ \\
\hline Leukemia or lymphoma & $78(18)$ & $660(18)$ & $1.05(0.82-1.34)$ \\
\hline Metastatic solid tumor & $56(13)$ & $449(12)$ & $1.19(0.90-1.58)$ \\
\hline HIV/AIDS & $10(2)$ & $66(2)$ & $1.30(0.70-2.44)$ \\
\hline \multicolumn{4}{|l|}{ Charlson composite score } \\
\hline $0-2$ & $223(53)$ & $2182(58)$ & Ref \\
\hline $3-5$ & $117(28)$ & $922(24)$ & $1.27(1.01-1.59)$ \\
\hline$>=6$ & $85(20)$ & $671(18)$ & $1.32(1.03-1.69)$ \\
\hline \multicolumn{4}{|l|}{ Case status $^{\mathrm{b}}$} \\
\hline HCFO/HCFA & $203(48)$ & $2332(62)$ & Ref \\
\hline CA or unknown & $57(13)$ & $597(16)$ & $1.07(0.79-1.43)$ \\
\hline CO/HCFA, indeterminate, or non- BJH HCFA & $165(39)$ & $846(22)$ & $2.17(1.76-2.66)$ \\
\hline Admitted from another healthcare facility & $109(26)$ & $1019(27)$ & $0.97(0.78-1.21)$ \\
\hline \multicolumn{4}{|l|}{ Number of inpatient admissions in previous 60 days } \\
\hline 0 & $200(47)$ & $2313(61)$ & Ref \\
\hline 1 & $150(35)$ & $1021(27)$ & $1.70(1.37-2.10)$ \\
\hline $2+$ & $75(18)$ & $441(12)$ & $1.96(1.50-2.55)$ \\
\hline \multicolumn{4}{|l|}{ Baseline laboratory data ${ }^{c}$} \\
\hline Low albumin at admission & $52(12)$ & $522(14)$ & $0.94(0.70-1.25)$ \\
\hline Low WBC at admission & $44(10)$ & $420(11)$ & $0.92(0.68-1.26)$ \\
\hline High WBC at admission & $236(56)$ & $2122(56)$ & $0.86(0.66-1.12)$ \\
\hline Low hemoglobin at admission & $182(43)$ & $1572(42)$ & $1.09(0.90-1.32)$ \\
\hline High creatinine at admission & $108(25)$ & $947(25)$ & $1.04(0.84-1.30)$ \\
\hline Low creatinine clearance & $218(51)$ & $1636(43)$ & $1.43(1.18-1.73)$ \\
\hline
\end{tabular}

$\mathrm{rCDI}=$ recurrent $\mathrm{C}$. difficile infection.

${ }^{a}$ Comorbidities diagnosed within previous 1 year (identified by ICD-9-CM diagnosis codes.

${ }^{b}$ HCFO = health care facility onset; HCFA = healthcare facility-associated; CA = community acquired; CO = community onset. Case she status for 6 patients' was unknown: 1 among those who developed $\mathrm{rCDI}$ and 5 among those who did not.

"The following threshold values defined "high" and "low" levels: albumin $<2.5 \mathrm{~g} / \mathrm{dL} ; \mathrm{WBC}$ low $<3.8^{*} 10^{3} / \mathrm{mm}^{3} ; \mathrm{WBC}$ high $>9.8^{*} 10^{3} / \mathrm{mm}^{3} ;$ hemoglobin $<10.0 \mathrm{~g} / \mathrm{dL}$; creatinine $>1.5 \mathrm{ug} / \mathrm{dL}$; creatinine clearance $<70 \mathrm{~mL} / \mathrm{min}$. $\mathrm{WBC}=$ white blood cells. 
Table 2 Processes of care at the onset of and treatment for the initial CDI hospitalization

\begin{tabular}{|c|c|c|c|}
\hline Patient characteristics & $\begin{array}{l}\text { Patients who developed rCDI } \\
\qquad(n=425)\end{array}$ & $\begin{array}{l}\text { Patients who did not develop rCDI } \\
\qquad(n=3775)\end{array}$ & $\begin{array}{c}\text { Hazard ratio } \\
95 \% \mathrm{Cl}\end{array}$ \\
\hline \multicolumn{4}{|l|}{ Laboratory results iCDI onset } \\
\hline Low albumin & $50(12)$ & $548(15)$ & $0.84(0.63-1.13)$ \\
\hline Low WBC & $64(15)$ & $635(17)$ & $0.99(0.82-1.20)$ \\
\hline High WBC & $247(58)$ & $2027(54)$ & $1.23(1.01-1.49)$ \\
\hline Low hemoglobin & $218(51)$ & $1985(53)$ & $0.96(0.79-1.16)$ \\
\hline High creatinine & $99(23)$ & $862(23)$ & $1.08(0.86-1.35)$ \\
\hline \multicolumn{4}{|l|}{ Relevant medications present at iCDI onset } \\
\hline Any antimicrobial & $314(74)$ & $2729(72)$ & $1.10(0.88-1.36)$ \\
\hline Low risk antimicrobial(s) ${ }^{a}$ & $95(22)$ & $1058(28)$ & $0.76(0.60-0.95)$ \\
\hline High risk antimicrobial(s) ${ }^{\mathrm{b}}$ & $174(41)$ & $1490(40)$ & $1.07(0.88-1.29)$ \\
\hline Fluoroquinolone & $120(28)$ & $861(23)$ & $1.29(1.05-1.60)$ \\
\hline IV vancomycin & $130(31)$ & $1321(35)$ & $0.86(0.70-1.05)$ \\
\hline Gastric acid suppressor, any & $310(73)$ & $2850(76)$ & $0.91(0.73-1.12)$ \\
\hline New gastric acid suppressor & $54(13)$ & $255(7)$ & $1.87(1.41-2.49)$ \\
\hline \multicolumn{4}{|l|}{ Relevant medications received following iCDI onset } \\
\hline Any antibiotic first dose after CDI & $278(65)$ & $1622(43)$ & $2.47(2.02-3.02)$ \\
\hline Low risk antimicrobial(s) first dose after $\mathrm{CDI}^{\mathrm{a}}$ & $141(33)$ & $710(19)$ & $2.09(1.71-2.56)$ \\
\hline High risk antimicrobial(s) first dose after $\mathrm{CDI}^{\mathrm{b}}$ & $150(35)$ & $714(19)$ & $2.30(1.89-2.81)$ \\
\hline Fluoroquinolone first dose after CDI & $124(29)$ & $703(19)$ & $1.69(1.37-2.09)$ \\
\hline IV vancomycin first dose after CDI & $115(27)$ & $337(12)$ & $2.61(2.11-3.23)$ \\
\hline \multicolumn{4}{|l|}{ Initial CDI treatment } \\
\hline Metronidazole alone & $323(76)$ & $2841(75)$ & Reference \\
\hline Oral vancomycin alone & $16(4)$ & $104(3)$ & $1.32(0.80-2.18)$ \\
\hline Metronidazole and oral vancomycin & $86(20)$ & $829(22)$ & $0.95(0.75-1.20)$ \\
\hline
\end{tabular}

$\mathrm{iCDI}=$ initial episode of $C$. difficile infection, $\mathrm{rCDI}=$ recurrent $C$. difficile infection.

a Low risk antimicrobials included aminoglycosides, betalactamase inhibitors, carbepenems, daptomycin, doxycycline, linezolid, macrolides, penicillinase inhibitors, rifampin, rifaximin, and tigecycline.

${ }^{\mathrm{b}}$ High risk antimicrobials included all cephalosporins, clindamycin, and penicillins.

the importance of time dependency of concomitant antimicrobials started after CDI treatment ended, when post-CDI treatment antimicrobials were modeled in a time independent fashion, their association with rCDI decreased significantly (Table 4).

\section{Discussion}

We have identified eight discrete independent risk factors for recurrent CDI. Although some characteristics, such as age, cannot be altered, several of them constitute modifiable exposures. New gastric acid suppression and concomitant antimicrobial exposures were associated with increased hazards of developing recurrent CDI. Reducing these exposures could potentially decrease the risk of recurrent CDI. This may serve as yet another reason for institutions to engage in aggressive antimicrobial stewardship programs.

Prior investigations have reported advanced age, chronic renal insufficiency, elevated white blood cell count, low serum albumin, use of PPI and H2RB, as well as continued use of systemic antimicrobials to be important risk factors for rCDI [7,10-13,22]. Our results are in general agreement with these prior data. Gastric acid suppressors have garnered a particular interest with respect to their impact on iCDI and rCDI incidence. Specific to recurrent disease, a recent meta-analysis substantiated this concern, finding a more-than doubling of the risk of $\mathrm{rCDI}$ in the setting of these drugs [7]. At the same time, it is unclear whether both PPIs and H2RBs are associated with the risk of rCDI, or whether one is a more likely culprit than the other. For example a meta-analysis by Kwok and colleagues implicated PPIs but not H2RBs in a 2-fold rise of rCDI incidence [23]. Similarly, Tleyjeh et al. in a meta-analysis of 33 studies focusing specifically on H2RB exposure reported a smaller, albeit still significant, association between receiving H2RBs and development of CDI [24]. Both metaanalyses suggested that gastric protection in conjunction with antibiotic administration carries a higher risk of CDI 
Table 3 Outcomes following initial CDI hospitalization

\begin{tabular}{|c|c|c|c|c|}
\hline Patient characteristics & $\begin{array}{c}\text { Patients who } \\
\text { developed rCDI } \\
(n=425)\end{array}$ & $\begin{array}{l}\text { Patients who did } \\
\text { not develop rCDI } \\
\quad(n=3775)\end{array}$ & $\begin{array}{l}\text { Hazard ratio } \\
\qquad 95 \% \mathrm{Cl}\end{array}$ & p-value \\
\hline Discharged to a healthcare facility & $130(31)$ & $1029(27)$ & $1.18(0.96-1.45)$ & 0.11 \\
\hline Inpatient readmission(s) before end of iCDI treatment & $48(11)$ & $241(6)$ & $1.76(1.30-2.37)$ & $<.001$ \\
\hline Inpatient readmission(s) after end of iCDI treatment & $126(30)$ & $857(23)$ & $1.31(1.07-1.62)$ & 0.01 \\
\hline
\end{tabular}

$\mathrm{iCDI}=$ initial episode of $C$. difficile infection, $\mathrm{rCDI}=$ recurrent $C$. difficile infection.

development than exposure to PPIs or H2RBs alone $[23,24]$. In our study, we examined gastric acid suppressors as a single category because our prior work, including preliminary analyses for this study (data not shown), has consistently found no difference in the associations between these two classes of medications and CDI $[17,18]$. Whether gastric acid suppression is truly an independent risk factor for CDI or a marker for patients at risk for CDI remains unknown [6].

A large body of evidence also ties concomitant use of non-CDI antimicrobials to an increased risk of a recurrence $[7,17,18,25]$. We found that high-risk antimicrobials raise the risk for rCDI, particularly when administered after the completion of iCDI treatment. We have also confirmed previous findings that link exposure to such specific antimicrobials as IV vancomycin and fluoroquinolones to the risk for CDI incidence $[17,18,26]$. The BI/NAP1/027 strain has been associated with fluoroquinolone exposures, and may be more likely to cause rCDI than other strains of C. difficile [27]. Consequently, it is possible that fluoroquinolone exposure is a marker for CDI specifically due to this strain. For IV vancomycin, however, this association may represent not a causal relationship, but rather a marker for higher illness severity and, thus, confounding by indication.

We were also able to demonstrate the importance of timing of antimicrobial exposure after the end of CDI treatment. When modeled as time dependent variables, high-risk antimicrobials, fluoroquinolones, and IV vancomycin were all associated with rCDI. When modeled as time independent variables, the hazards of rCDI associated with high-risk antimicrobials dropped from

Table 4 Cox proportional hazards multivariable model examining risk factors for recurrent CDI

\begin{tabular}{|c|c|c|c|c|}
\hline \multirow{2}{*}{$\begin{array}{l}\text { Patient characteristics } \\
\text { Risk factor }\end{array}$} & \multicolumn{2}{|c|}{$\begin{array}{l}\text { Antimicrobials after iCDI treatment modeled } \\
\text { as time dependent variables }\end{array}$} & \multicolumn{2}{|c|}{$\begin{array}{c}\text { Antimicrobials after iCDI treatment modeled } \\
\text { as time independent variables }\end{array}$} \\
\hline & Hazard ratio & $95 \% \mathrm{Cl}$ & Hazard ratio & $95 \% \mathrm{Cl}$ \\
\hline \multicolumn{5}{|l|}{ At admission to the hospital } \\
\hline \multicolumn{5}{|l|}{ CDI case status } \\
\hline $\mathrm{HOCDI}$ & Ref & & Ref & \\
\hline COHCFA CDI & 1.80 & $1.41-2.29$ & 1.78 & $1.39-2.27$ \\
\hline CA CDI & 1.30 & $0.95-1.80$ & 1.25 & $0.91-1.72$ \\
\hline \multicolumn{5}{|l|}{ Number of hospitalizations in previous 60 days } \\
\hline None & Ref & & & \\
\hline 1 & 1.25 & $0.97-1.61$ & 1.27 & $0.99-1.64$ \\
\hline$>1$ & 1.40 & $1.04-1.89$ & 1.46 & $1.08-1.96$ \\
\hline Age (per 1 year) & 1.01 & $1.00-1.02$ & 1.01 & $1.00-1.02$ \\
\hline \multicolumn{5}{|l|}{ At the onset or during treatment of iCDI } \\
\hline Gastric acid suppression & 1.36 & $1.00-1.85$ & 1.40 & $1.03-1.90$ \\
\hline Cumulative fluoroquinolone exposure $^{a}$ & 1.24 & $1.09-1.41$ & 1.42 & $1.25-1.61$ \\
\hline \multicolumn{5}{|l|}{ Following completion of iCDI treatment } \\
\hline High risk antimicrobial ${ }^{b}$ & $2.95^{c}$ & $2.25-3.86$ & 1.86 & $1.42-2.42$ \\
\hline Fluoroquinolone & $1.56^{\mathrm{c}}$ & $1.63-2.08$ & 0.86 & $0.64-1.15$ \\
\hline IV vancomycin & $1.45^{c}$ & $1.09-1.92$ & 1.05 & $0.80-1.39$ \\
\hline
\end{tabular}

$\mathrm{iCDI}=$ initial episode of $C$. difficile infection.

${ }^{a}$ Cumulative fluoroquinolone exposure was modeled as a three node spline.

${ }^{\mathrm{b}}$ High risk antimicrobials included all cephalosporins, clindamycin, and penicillins.

'Exposure at any time $t$. 
2.95 (2.25-3.86) to 1.86 (1.42-2.42), and fluoroquinolones and IV vancomycin were no longer associated with rCDI. Intuitively, this makes sense. An antimicrobial should not increase the risk of rCDI after CDI treatment has ended until the patient is exposed to the antimicrobial. Not modeling antimicrobials as time dependent variables after CDI treatment has ended dilutes the association with rCDI, since the days not on these drugs are included in the model.

A direct relationship between CO-HCFA status and iCDI and rCDI development is a newer finding [15]. Namely, the CO-HCFA designation of the iCDI episode is associated with at least a $25 \%$ and as much as a 2 -fold increase in the risk of rCDI. A likely mechanism relates to the fact that CO-HCFA defines a population of patients who is likely sicker as evident by recent hospitalizations, and more likely to be exposed to antimicrobials. However, CO-HCFA CDI remained an independent risk factor when controlling for recent hospitalizations.

It is worth noting that the recurrence rate we observed in the current study is at the lower end of what has been reported previously. For example, a recent meta-analysis by Garey et al. examined the literature on risk factors for rCDI [7]. In the 12 studies meeting the inclusion criteria, the rates of recurrence ranged from $13 \%$ to $50 \%$. More current data from randomized controlled trials suggest that CDI is likely to recur in approximately $25 \%$ of the patients treated for iCDI with vancomycin $[8,9]$. A potential explanation for the lower rCDI rate in our study compared to others is how cases of CDI were identified. Most stools submitted for $C$. difficile testing at the $\mathrm{BJH}$ microbiology laboratory come from inpatients and the emergency room. A minority of specimens come from outpatients or affiliated skilled nursing facilities. It is likely that milder cases of rCDI were missed because the patient did not require care in an emergency room or need to be admitted. Therefore, the rCDI in this study may consist of more clinically important episodes, occurring in sicker patients, many of whom required an admission or evaluation in the emergency department.

It is possible that patients who resided outside the St. Louis metropolitan area would not be likely to return to $\mathrm{BJH}$ for testing for a recurrence. To examine the impact of this potential loss to follow up, we performed a sensitivity analysis of rCDI risk factors by excluding all patients who resided beyond the greater St. Louis postal code. After excluding the 1230 (31.1\%) patients with iCDI who met this criterion, the rCDI risk factors and their hazard ratios did not change appreciably (data not shown). This suggested that our results were not biased by including these patients.

Conversely, rCDI in randomized trials may be subject to a detection bias. Patients in trials are prospectively monitored for recurrent diarrhea and instructed to seek testing if it occurs. Even if the patient's symptoms are not from CDI, the person may test positive for CDI as many patients continue to shed $C$. difficile in stool after cessation of CDI treatment [28].

Our study has some limitations. As a retrospective observational study it is prone to several forms of bias, most notably a selection bias. To mitigate this, we enrolled all consecutive patients meeting our enrollment criteria. To avoid misclassification of the main outcome variable, we applied a stringent case definition to CDI, which included a positive toxin assay. Although confounding is an issue with observational data, we adjusted for all the available relevant potential confounders in the regression model. However, the possibility of residual confounding remains. The biggest limitation, however, is its generalizability, since the data reflected patients and treatment patterns at an urban academic medical center with a large referral base, and may not have mirrored those of institutions with different characteristics or patients with iCDI diagnosed and managed completely in the outpatient setting. Additionally, many of the patients who resided outside the St. Louis metropolitan area may not have had their specimens retested at the BJH laboratory. After excluding these patients from the analysis as part of a sensitivity analysis, neither the rCDI hazard ratios nor the rCDI risk factors were majorly impacted in the overall cohort.

\section{Conclusion}

In summary, we have demonstrated that a number of modifiable factors exist whose presence raises the risk for developing rCDI. Avoiding such exposures as nonCDI antimicrobial treatment and gastric acid suppressors may go a long way toward attenuating the burden of rCDI. On the other hand recognizing CO-HCFA and advanced age as predispositions to rCDI should serve patients and clinicians well by highlighting the importance of targeting these populations for more aggressive prevention efforts.

\section{Competing interests}

MDZ reports that she has received research support from Optimer, and research and consulting support from ViroPharma and from Cubist. ERD reports that he has performed research for Viropharma and Merck and has served as a consultant for Merck, Becton-Dickinson, Optimer, Meridian, and Steris. All other authors report no conflicts of interest relevant to this article.

\section{Authors' contributions}

MDZ conceived of the study, participated in its design, interpretation of the analyses and drafting of the manuscript. KR participated in carrying out and coordinating the analyses and drafting of the manuscript. MO participated in carrying out the analyses and their interpretation, as well as drafting of the manuscript. $Y Y$ was responsible for the integrity of the statistical analyses and for carrying them out, as well as drafting the manuscript. ERD participated in the design and oversight of the study, as well as drafting the manuscript. All authors read and approved the final manuscript. 


\section{Acknowledgements}

This study was supported by Cubist Pharmaceuticals, Inc., San Diego, CA. The funder had no role in study design, analyses, data interpretation, or in the preparation or submission of the manuscript. Although the funder was given the opportunity to review the manuscript prior to submission, all of the editorial decisions resided with the authors. No one other than the listed authors contributed to the study.

\section{Disclosure}

Study supported by Cubist Pharmaceuticals, Inc., San Diego, CA.

The data in the manuscript were presented in part as a podium presentation at ID week 2012 in San Diego, CA.

\section{Author details}

${ }^{1}$ University of Massachusetts, Amherst, MA, USA. ${ }^{2}$ EviMed Research Group, LLC, PO Box 303, Goshen, MA, USA. ${ }^{3}$ Washington University School of Medicine, 660 S. Euclid Ave, St. Louis, MO 63110, USA.

Received: 3 February 2014 Accepted: 26 May 2014

Published: 4 June 2014

\section{References}

1. Pépin J, Valiquette L, Alary ME, Villemure P, Pelletier A, Forget K, Pépin K, Chouinard D: Clostridium difficile-associated diarrhea in a region of Quebec from 1991 to 2003: a changing pattern of disease severity. CMAJ 2004, 171:466-472.

2. McDonald LC, Owings M, Jernigan DB: Clostridium difficile infection in patients discharged from US short-stay hospitals, 1996-2003. Emerg Infect Dis 2006, 12:409-415.

3. Zilberberg MD, Shorr AF, Kollef MH: Increase in adult Clostridium difficilerelated hospitalizations and case-fatality rate, United States, 2000-2005. Emerg Infect Dis 2008, 14:929-931.

4. Lucado, J. (Social \& Scientific Systems), Gould, C. (CDC), and Elixhauser, A. (AHRQ): Clostridium difficile Infections (CDI) in Hospital Stays, 2009. HCUP Statistical Brief \#124. 2012, Agency for Healthcare Research and Quality, Rockville, MD. http://www.hcup-us.ahrq.gov/reports/statbriefs/sb124.jsp.

5. Clements AC, Magalhaes RJ, Tatem AJ, Paterson DL, Riley TV: Clostridium difficile PCR ribotype 027: assessing the risks of further worldwide spread. Lancet Incect Dis 2010, 10:395-404.

6. Cohen SH, Gerding DN, Johnson S, Kelly CP, Loo VG, McDonald LC, Pepin J, Wilcox $\mathrm{MH}$ : Clinical practice guidelines for Clostridium difficile infection in adults: 2010 update by the society for healthcare epidemiology of America (SHEA) and the infectious diseases society of America (IDSA) Infect Control Hosp Epidemiol 2010, 31(5):431-455.

7. Garey KW, Sethi S, Yadav Y, DuPont HL: Meta-analysis to assess risk factors for recurrent Clostridium difficile infection. J Hosp Infect 2008, 70:298-304.

8. Louie TJ, Miller MA, Mullane KM, Weiss K, Lentnek A, Golan Y, Gorbach S, Sears P, Shue YK, OPT-80-003 Clinical Study Group: Fidaxomicin versus vancomycin for Clostridium difficile infection. N Engl J Med 2011, 364:422-431.

9. Cornely OA, Crook DW, Esposito R, Poirier A, Somero MS, Weiss K, Sears P, Gorbach S, OPT-80-004 Clinical Study Group: Fidaxomicin versus vancomycin for infection with Clostridium difficile in Europe, Canada, and the USA: a double-blind, non-inferiority, randomised controlled trial. Lancet Infect Dis 2012, 12:281-289.

10. Fekety R, McFarland LV, Surawicz CM, Greenberg RN, Elmer GW, Mulligan ME: Recurrent Clostridium difficile diarrhea: characteristics of and risk factors for patients enrolled in a prospective, randomized, double-blinded trial. Clin Infect Dis 1997, 24:324-333.

11. McFarland LV, Surawicz CM, Rubin M, Fekety R, Elmer GW, Greenberg RN: Recurrent Clostridium difficile: epidemiology and clinical characteristics. Infect Control Hosp Epidemiol 1999, 20:43-50.

12. Cadle RM, Mansouri MD, Logan N, Kudva DR, Musher DM: Association of proton-pump inhibitors with outcomes in Clostridium difficile colitis. Am J Health Syst Pharm 2007, 64:2359-2363.

13. Kim JW, Lee KL, Jeong JB, Kim BG, Shin S, Kim JS, Jung H, Son IS: Proton pump inhibitors as a risk factor for recurrence of Clostridium-difficileassociated diarrhea. World J Gastroenterol 2010, 16:3573-3577.

14. Zilberberg MD, Tabak YP, Sievert DM, Derby KG, Johannes RS, Sun X, McDonald LC: Using electronic health information to risk-stratify rates of
Clostridium difficile infection in US hospitals. Infect Control Hosp Epidemiol 2011, 32:649-655.

15. Eyre DW, Walker AS, Wylie D, Dingle KE, Griffiths D, Finney J, O'Connor L, Vaughan A, Crook DW, Wilcox MH, Peto TE: Predictors of first recurrence of Clostridium difficile infection: implications for initial management. Clin Infect Dis 2012, 55:S77-S87.

16. McDonald LC, Coignard B, Dubberke E, Song X, Horan T, Kutty PK: Recommendations for surveillance of Clostridium difficile-associated disease. Infect Control Hosp Epidemiol 2007, 28:140-145.

17. Dubberke ER, Reske KA, Yan Y, Olsen MA, McDonald LC, Fraser VJ: Clostridium difficile-associated disease in a setting of endemicity: identification of novel risk factors. Clin Infect Dis 2007, 45:1543-1549.

18. Dubberke ER, Yan Y, Reske KA, Butler AM, Doherty J, Pham V, Fraser VJ: Development and validation of a Clostridium difficile infection risk prediction model. Infect Control Hosp Epidemiol 2011, 32:360-366.

19. Hosmer DW, Lemeshow S: In Applied Logistic Regression. 2nd edition. Edited by New York, NY. New York, NY: John Wiley and Sons, Inc; 2000.

20. Harrell FE Jr, Margolis PA, Gove S, Mason KE, Mulholland EK, Lehmann D, Muhe L, Gatchallan S, Eichenwald HF: Development of a clinical prediction model for an ordinal outcome: the World Health Organization Multicentre Study of Clinical Signs and Etiological agents of Pneumonia, Sepsis and Meningitis in Young Infants. WHO/ARI Young Infant Multicentre Study Group. Stat Med 1998, 17:909-944.

21. SAS: Copyright (c) 2002-2010. Cary, NC, USA: SAS Institute Inc;

22. Kim YG, Graham DY, Jang BI: Proton pump inhibitor use and recurrent Clostridium difficile-associated disease: a case-control analysis matched by propensity score. J Clin Gastroenterol 2012, 46:397-400.

23. Kwok CS, Arthur AK, Anibueze Cl, Singh S, Cavallazzi R, Loke YK: Risk of Clostridium difficile infection with acid suppressing drugs and antibiotics: meta-analysis. Am J Gastroenetrol 2012, 107:1011-1019.

24. Tleyjeh IM, Abdulhak AB, Riaz M, Garbati MA, Al-Tannir M, Alasmari FA, Alghamdi M, Khan AR, Erwin PJ, Sutton AJ, Baddour LM: The association between histamine 2 receptor antagonist use and Clostridium difficile infection: a systematic review and meta-analysis. PLOS One 2013, 8:e56498.

25. Mullane KM, Miller MA, Weiss K, Lentnek A, Golan Y, Sears PS, Shue YK, Louie TJ, Gorbach SL: Efficacy of fidaxomicin versus vancomycin as therapy for Clostridium difficile infection in individuals taking concomitant antibiotics for other concurrent infections. Clin Infect Dis 2011, 53:440-447.

26. Pépin J, Saheb N, Coulombe MA, Alary ME, Corriveau MP, Authier S, Leblanc M, Rivard G, Bettez M, Primeau V, Nguyen M, Jacob CE, Lanthier L: Emergence of fluoroquinolones as the predominant risk factor for Clostridium difficile-associated diarrhea: a cohort study during an epidemic in Quebec. Clin Infect Dis 2005, 41:1254-1260.

27. Petrella LA, Sambol SP, Chenknis A, Nagaro K, Kean Y, Sears PS, Babakhani F, Johnson S, Gerding DN: Decreased cure and increased recurrence rates for Clostridium difficile Infection caused by the epidemic C. difficile BI strain. Clin Infect Dis 2012, [Epub ahead of print].

28. Sethi AK, Al-Nassir WN, Nerandzic MM, Bobulsky GS, Donskey CJ: Persistence of skin contamination and environmental shedding of Clostridium difficile during and after treatment of $C$. difficile infection. Infect Control Hosp Epidemiol 2010, 31:21-27.

\section{doi:10.1186/1471-2334-14-306}

Cite this article as: Zilberberg et al:: Risk factors for recurrent Clostridium difficile infection (CDI) hospitalization among hospitalized patients with an initial CDI episode: a retrospective cohort study. BMC Infectious Diseases 2014 14:306. 